\title{
A patient with microhematuria and proteinuria: Do we still need electronmicroscopy?
}

\author{
M. J. Mihatsch, H. A. Bock ${ }^{1}$ and F. Gudat \\ Institut for Pathology, University Basel and ${ }^{1}$ Division of Nephrology, Cantonal Hospital, Basel, Switzerland
}

A 47-year-old white male was referred to the medical service for newly discovered hypercholesterolemia. He previously had been seen by the ENT service for progressive bilateral hearing loss for four years, which was determined to be of the inner ear type.

There was no relevant family history: specifically two sisters and his 79-year-old father were reported to be in good health. His 78-year-old mother had a history of gout but was otherwise well.

His personal history included an infectious mononucleosis at the age of 19 which apparently had been accompanied by 'nephritis'. There was again an episode of 'nephritis' including macrohaematuria at the age of 22 , possibly related to a respiratory infection for which he had been prophylactically treated with penicillin for 4 years. An occupational health screening at the age of 34 had revealed proteinuria which was not further investigated at that time. His physical examination was unremarkable except for elevated blood pressure $(150 / 100 \mathrm{mmHg})$; in particular, there was no oedema. He took no medication or drugs, did not smoke and drank approximately $4 \mathrm{dl}$ of wine per day.

Routine work-up showed a serum sodium of $142 \mathrm{mmol} / \mathrm{l}$, potassium $4.0 \mathrm{mmol} / 1$, creatinine $103 \mu \mathrm{mol} / 1$, urea $7.0 \mathrm{mmol} / 1$, cholesterol $7.94 \mathrm{mmol} / 1$, triglycerides $1.68 \mathrm{mmol} / 1$, albumin $36 \mathrm{~g} / 1$ and protein $59 \mathrm{~g} / \mathrm{l}$. His haemoglobin was $16.4 \mathrm{~g} / \mathrm{dl}$, leukocytes $9170 / \mathrm{mm}^{3}$, platelets $269000 / \mathrm{mm}^{3}$. Liver enzymes, bilirubin and blood sugar profile were normal, as were serum electrophoresis, serum complement and antistreptolysin titre. Antinuclear antibodies were negative.

The urinary sediment showed +++ proteinuria, $>40$ dysmorphic erythrocytes/HPF, 0,3 leukocytes/ $\mathrm{HPF}$, hyaline and a few granular casts as well as lipid droplets and some oval fat bodies. 24-h proteinuria was $5.0 \mathrm{~g} / \mathrm{d}$ and creatinine clearance $86 \mathrm{ml} / \mathrm{min}$. BenceJones protein in urine was negative. Abdominal ultrasound showed two normal sized kidneys and was otherwise normal.

Correspondence and offprint requests to: M. J. Mihatsch, Institute for Pathology, University Basel, Basel, Switzerland.

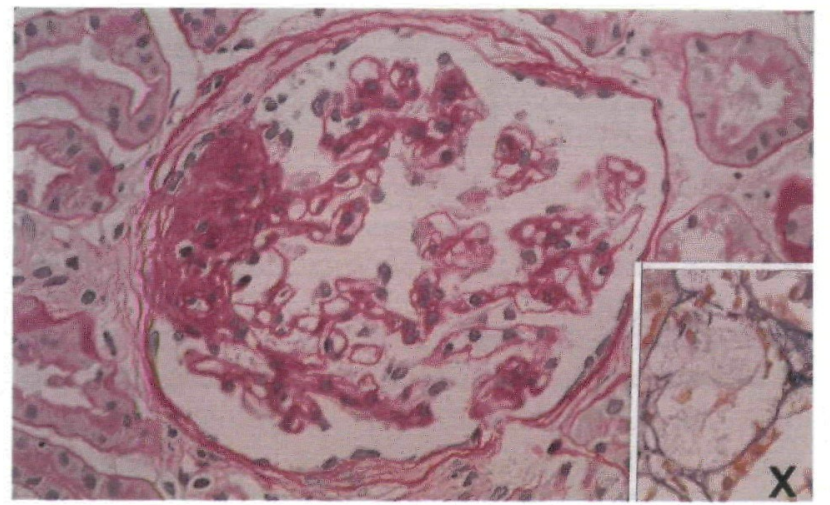

Fig. 1. Segmental glomerular sclerosis. Inset: group of interstitial foam cells.

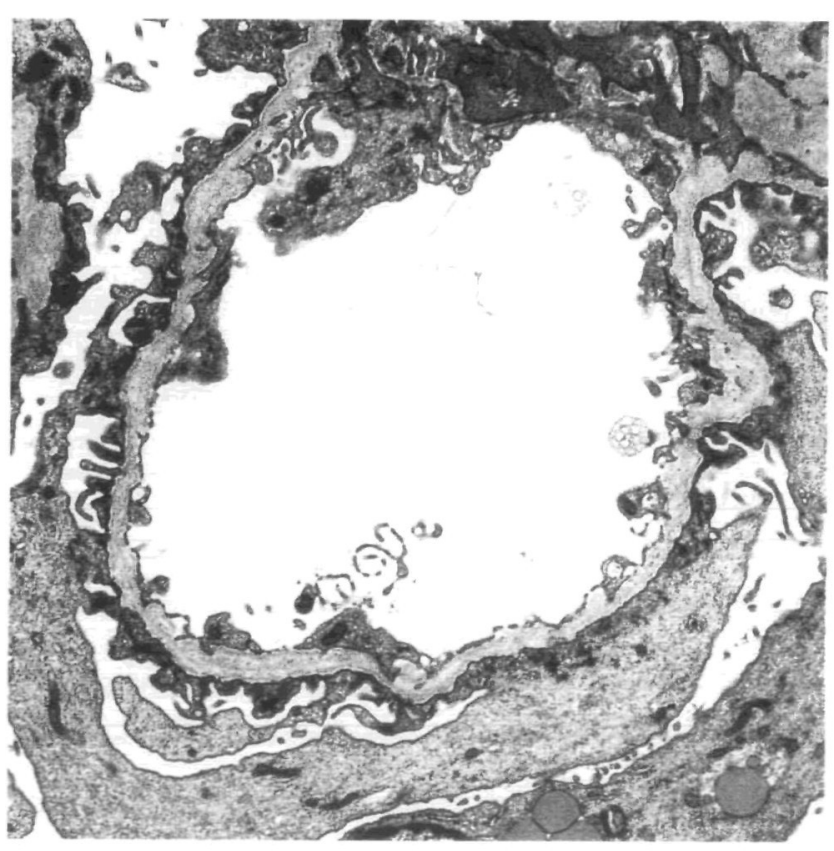

Fig. 2. Peripheral glomerular capillary loop: the basement membrane shows thinning and splitting of the lamina densa. 


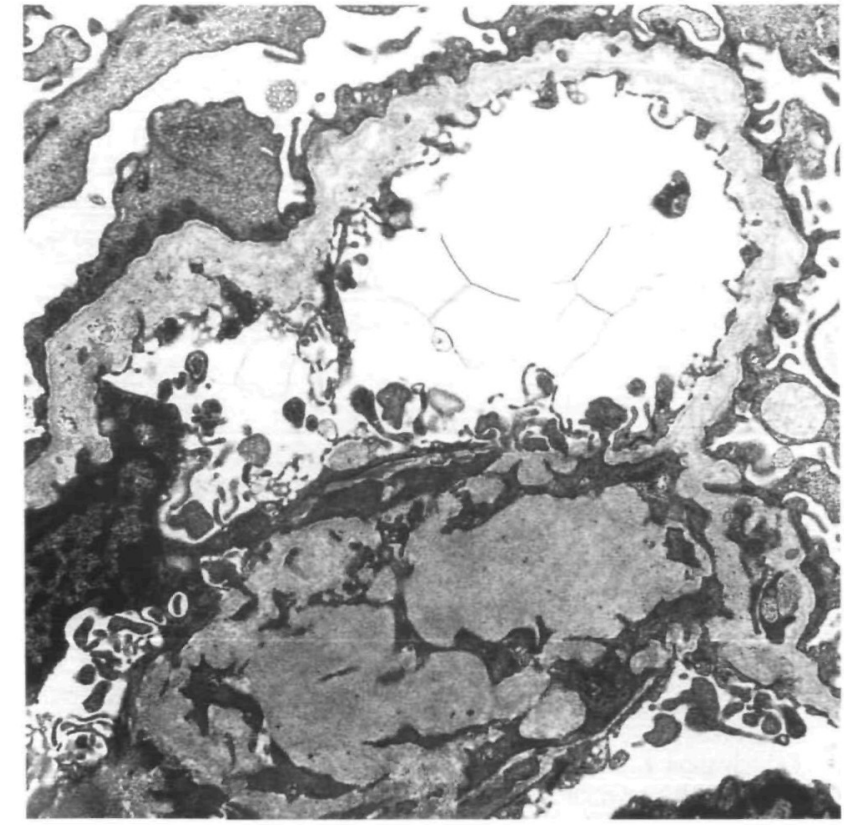

Fig. 3. Peripheral glomerular capillary loop: the basement shows focal thinning and pronounced irregular thickening. The lamina densa is split and fragmented.

\section{Morphology of kidney biopsy}

By light microscopy eleven glomeruli were found of which three showed segmental sclerosis (Figure 1). The arterioles exhibited medium severe arteriolar hylinosis. In the interstitial space groups of foam cells were present (Figure 1). Also some of the tubular cells exhibited a foam transformation. A minimal interstitial fibrosis with tubular atrophy was present. Foetal-like glomeruli were not found.

Immunohistological investigation (IgG, IgM, IgA,
C3, C4, fibrin) showed minimal segmental deposits of IgG, IgA and C3 in glomeruli with segmental sclerosis.

Two glomeruli investigated by electron microscopy exhibited thinning and thickening of the basement membrane with a lamellation and fragmentation of the lamina densa in all loops (Figures 2 and 3).

The EM findings were interpreted as characteristic of Alport's syndrome.

\section{This case is of interest for several reasons (teaching point):}

Is electron microscopy still necessary in so-called clearcut diagnostic situations? In the present case, light microscopy showed segmental sclerosis, immunohistology was compatible with this finding. So, the diagnosis of segmental glomerular sclerosis could be accepted as sufficiently documented. However, electron microscopy showed lesions typical of Alport's syndrome. Therefore, we still believe that electron microscopy is essential for the definite diagnosis of renal biopsies of native kidneys. Our studies of hundred consecutive renal biopsies have shown that electron microscopy contributes clinically relevant additional information in about $30 \%$ of cases [1]. The most important point is that the combined use of light microscopy, immunohistology and electron microscopy results in nearly all cases in an unequivocal diagnosis which forms a sound basis for the further management of the patient.

Interstitial foam cells are usually thought to be only present in cases of long standing nephrotic syndrome. The primary clinical information: 'glomerular hematuria and proteinuria without nephrotic syndrome' raised our suspicion of Alport's syndrome due to the presence of foam cells. Foam cells are commonly found in cases

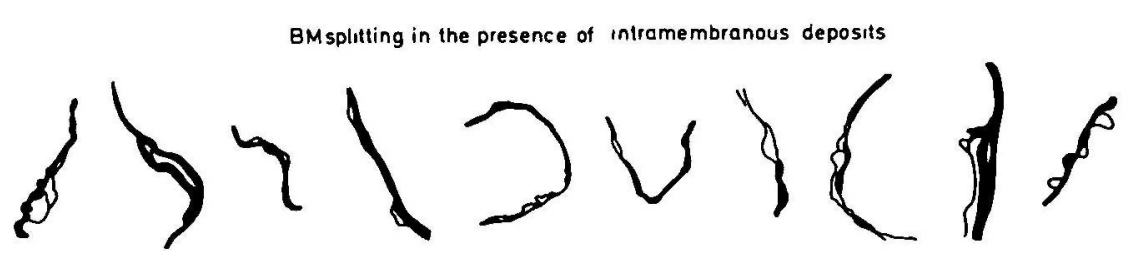

BM lamellation and fragmentation in Alport's syndrome
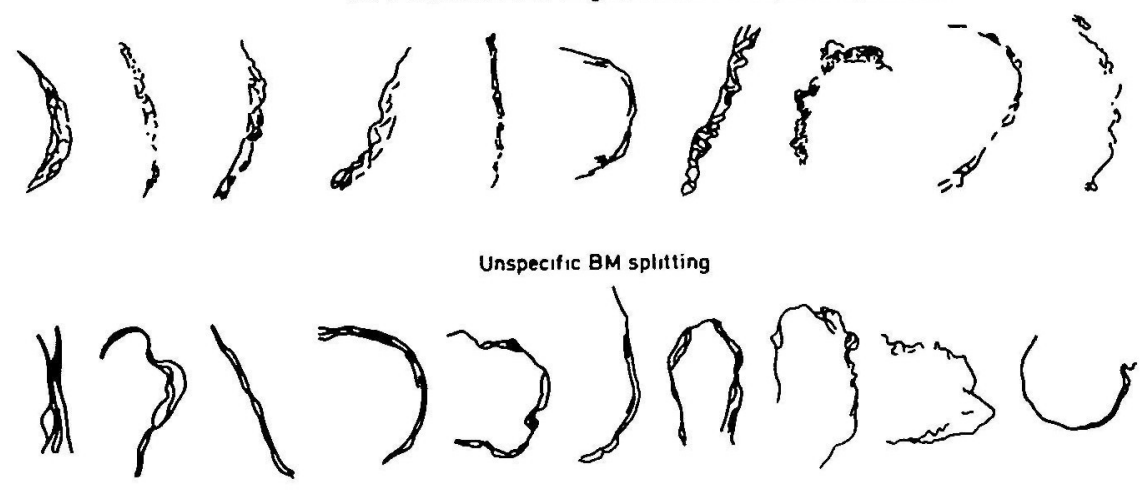

Fig. 4. Differential diagnosis of basement membrane splitting [4]. 
of nephrotic syndrome and in Alport's syndrome. In Alport's syndrome foam cells are present without nephrotic syndrome and are far more common than in glomerulonephritis [1,4]. About $40 \%$ of cases of Alport's syndrome are accompanied by foam cells whereas only $6 \%$ of biopsies with glomerulonephritis. Thus, the presence of foam cells without long standing nephrotic syndrome should always prompt electron microscopic investigation. In our patients severe proteinuria ( $>5 \mathrm{~g} /$ day) with hypercholesterinemia $(\sim 8 \mathrm{mmol} / \mathrm{l})$ and lipiduria could, however, explain the presence of foam cells even in the absence of full blown nephrotic syndrome or Alport's syndrome.

Since the publication of Spear and Slusser [2] electron microscopic findings were considered the golden standard for the diagnosis of Alport's syndrome. The characteristic features are basement membrane thinning and thickening accompanied by a lamellation, reticulation and fragmentation of the lamina densa as found in the present case.

Since the beginning, it was, however, clear that also in other diseases basement changes may be found which may be confused with Alport's basement membrane changes (Figure 4). Recent studies have shown that patients with Alport's syndrome have a mutation of the Alpha 5 chain of collagen 4 (COL 4 A5) which is located on the long arm of the $\mathrm{X}$ chromosome [3].

Therefore, all cases with so-called typical Alport lesions by electron microscopy should be confirmed in the future by chromosomal studies. This time consuming and expensive study is justified since the diagnosis of Alport's syndrome has extensive implications for the patient e.g. life insurance, family planning etc.

Is the trias of deafness, hematuria and proteinuria proof of Alport's syndrome?

\section{References}

1. Mihatsch MJ, Zollinger HU. Ist eine elektronenmikroskopische Untersuchung von Nierenbiopsien klinisch nötig, wünschenswert, unnötig? Med Klin (1978); 73: 389-394

2. Spear GS, Slusser RJ. Alport's syndrome: Emphasizing electron microscopic studies of the glomerulus. Am J Pathol 1972; 69: 213-222

3. Hostikka SL, Eddy RL, Byers MG, Hoyhtya M, Shows TB, Tryggvason $\mathrm{K}$. Identification of a distinct type IV collagen alpha chain with restricted kidney distribution and assignment of its gene to the locus of $\mathrm{X}$ chromosome-linked Alport syndrome. Proc Natl Acad Sci USA 1990; 87: 1606-1610

4. Zollinger HU, Mihatsch MJ. Renal pathology in biopsy. Springer Verlag, Berlin, Heidelberg, New York: 1978 